\title{
Supporting Informantion
}

\section{Multicomponent spinel metal oxide nanocomposites as high-performance bifunctional catalysts in $\mathrm{Zn}$-air batteries}

Shiyin Li, ${ }^{\text {a,1 }}$ Xuyan Zhou, ${ }^{\mathrm{a}, 1}$ Gang Fang, ${ }^{\mathrm{a}, 1}$ Guoqiang Xie, ${ }^{\mathrm{a}}$ Xingjun Liu, ${ }^{\mathrm{a}, \mathrm{b}}$, Xi Lin*a and Hua-Jun Qiu*a

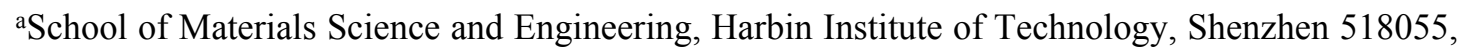

China

Email: qiuhuajun@hit.edu.cn (H.J.Q.); linxi@hit.edu.cn (X.L.)

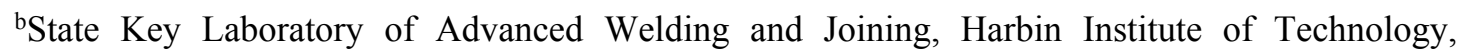
Shenzhen 518055, China

${ }^{1}$ Contribute equally to this work. 


\section{Experimental sections}

Materials preparation. The precursor alloy ribbons with atomic ratios of $\mathrm{Al}_{96} \mathrm{Co}_{4}$, $\mathrm{Al}_{96} \mathrm{Fe}_{2} \mathrm{Co}_{2}, \mathrm{Al}_{96} \mathrm{Mn}_{4}, \mathrm{Al}_{96} \mathrm{Co}_{2} \mathrm{Mn}_{2}, \mathrm{Al}_{95.5} \mathrm{Fe}_{1.5} \mathrm{Co}_{1.5} \mathrm{Mn}_{1.5}, \mathrm{Al}_{96} \mathrm{Fe}_{1} \mathrm{Co}_{1} \mathrm{Mn}_{1} \mathrm{X}_{1}$ (X: Ni, $\mathrm{Cr}, \mathrm{Nb}, \mathrm{V}$ ) and $\mathrm{Al}_{95.5} \mathrm{Fe}_{1.5} \mathrm{Co}_{1.5} \mathrm{Ni}_{1.5}$ were prepared by melting pure melts (>99.9 wt.\%) under Ar protection and followed by melt-spinning. The tangent speed of the $\mathrm{Cu}$ roller is $30 \mathrm{~m} / \mathrm{s}$. Chemical dealloying in a $0.5 \mathrm{M} \mathrm{NaOH}$ solution followed by annealing in air at $300{ }^{\circ} \mathrm{C}$ for $2 \mathrm{~h}$ was performed to synthesize the nanoporous multicomponent metal oxides nanocomposites. The catalyst inks were prepared by sonicating a mixture of $3 \mathrm{mg}$ oxides, $4.0 \mathrm{mg}$ carbon nanotubes, $300 \mu \mathrm{L}$ isopropanol, and $100 \mu \mathrm{L}$ Nafion $(0.5 \mathrm{wt} . \%)$ for $\sim 20 \mathrm{~min}$. The catalyst ink $(\sim 4 \mu \mathrm{L})$ was dropped on a glassy carbon electrode and naturally dried for the electrochemical tests. For comparison, the commercial Pt/C catalyst (20 wt.\%, Johnson-Matthey) and $\mathrm{IrO}_{2}$ (Aladdin) were also studied for comparison.

Materials Characterization: Sample characterization was performed on a JEOL ARM-200F transmission electron microscope (TEM) equipped with an EDS detector, a scanning electron microscope (SEM, HITACHI S-4700) also equipped with EDS, an X-ray diffraction (XRD) diffractometer using $\mathrm{Cu} \mathrm{K \alpha}$ radiation (Rigaku D/Max 2500), and an X-ray photoelectron spectroscopy (XPS, ESCALAB 250).

Electrochemical ORR and OER tests. Electrochemical tests were carried out by using an electrochemical workstation (CHI 660e) and a three-electrode system with the modified glassy carbon electrode as working electrode, a Pt foil as the counter electrode and $\mathrm{Ag} / \mathrm{AgCl}$ as the reference electrode. All measurements were conducted in pure $\mathrm{O}_{2}$ saturated solution $(0.1 \mathrm{M}$ and $1.0 \mathrm{M} \mathrm{KOH}$ aqueous solutions for ORR and OER, respectively). Linear scan voltammetry and cyclic voltammetry were conducted at a scan rate of $5 \mathrm{mV} \mathrm{s}^{-1}$. The current density was normalized to the geometrical area and the measured potentials vs $\mathrm{Ag} / \mathrm{AgCl}$ were converted to a reversible hydrogen electrode (RHE) scale according to the Nernst equation $\left(\mathrm{E}_{\mathrm{RHE}}=\mathrm{E}_{\mathrm{Ag} / \mathrm{AgCl}}+0.059 \mathrm{pH}+\right.$ 0.197); the overpotential $(\eta)$ for OER was calculated according to the following formula: $\eta(\mathrm{V})=\mathrm{E}_{\mathrm{RHE}}-1.23 \mathrm{~V}$. 
Battery tests. The $\mathrm{Zn}$-air batteries were assembled with the catalyst modified carbon cloth (catalyst loading: $\sim 0.5 \mathrm{mg} \mathrm{cm}^{-2}$ ) as the air cathode, a Zn foil (0.10 mm thickness) as the anode, and $6 \mathrm{M} \mathrm{KOH}$ aqueous solution as the electrolyte.

\section{Density functional theory calculation :}

The spin-polarized density functional theory (DFT) computations were performed using the Vienna ab initio Simulation Package (VASP). We used the Perdew-Burke-Ernzerhof (PBE) functional and the projector augmented wave (PAW) pseudo-potentials. The energy cutoff was $450 \mathrm{eV}$. The supercells of $\mathrm{Mn}_{3} \mathrm{O}_{4}$ and $\mathrm{Co}_{3} \mathrm{O}_{4}$ each contained 84 atoms. The thickness of vacuum along the z-direction was $13 \AA$ to minimize the artificial interactions among the supercell images. The Brillouin zone of each supercell was sampled using a $2 \times 2 \times 1$ uniform k-point mesh. All the atomic structures were fully relaxed until the forces on each atom being less than $0.02 \mathrm{eV} / \AA$ and the energy variation between two iterations being less than $1 \times 10^{-5} \mathrm{eV}$. During the structural relaxation, the bottom layer metal atoms were constrained to mimic the structure of a semi-infinite solid. The Grimme's semiempirical DFT-D3 scheme of the dispersion correction was adopted to describe the van der Waals (vdW) interactions in these layered systems.

The overpotentials that described the catalytic activities for ORR and OER were calculated by the standard hydrogen electrode method. ${ }^{1}$ The overall ORR reaction we considered in this work was

$$
\mathrm{O}_{2}(\mathrm{~g})+2 \mathrm{H}_{2} \mathrm{O}(\mathrm{l})+4 \mathrm{e}^{-} \rightarrow 4 \mathrm{OH}^{-}
$$

We considered the four-electron reaction pathway in alkaline media, with the ORR reaction pathway as follow:

$$
\begin{gathered}
*+\mathrm{O}_{2}(\mathrm{~g})+\mathrm{H}_{2} \mathrm{O}(\mathrm{l})+\mathrm{e}^{-} \rightarrow \mathrm{OOH}^{*}+\mathrm{OH}^{-} \\
\mathrm{OOH}^{*}+\mathrm{e}^{-} \rightarrow \mathrm{O}^{*}+\mathrm{OH}^{-} \\
\mathrm{O}^{*}+\mathrm{H} 2 \mathrm{O}(\mathrm{l})+\mathrm{e}^{-} \rightarrow \mathrm{OH}^{*}+\mathrm{OH}^{-} \\
\mathrm{OH}^{*}+\mathrm{e}^{-} \rightarrow *+\mathrm{OH}^{-}
\end{gathered}
$$


where $*$ represented the catalyst surface. The $\mathrm{OOH}^{*}, \mathrm{O}^{*}$ and $\mathrm{OH}^{*}$ species were oxygenated intermediates. Note that the reaction pathway of OER was the inverse process of ORR. In this work, the Gibbs free energy of each step was given by

$$
\Delta G=\Delta E_{\mathrm{DFT}}+\Delta Z P E-T \Delta S-e U
$$

where $\triangle E_{\mathrm{DFT}}, \triangle Z P E$ and $\Delta S$ were the energy changes in the DFT total energy, zero-point energy and entropy from the initial state to the final state, respectively.

Here, the values of $\mathrm{H}_{2} \mathrm{O}$ and $\mathrm{H}_{2}$ molecules were obtained from the NIST-JANAF thermodynamics tables and the vibrational frequencies of $\mathrm{OOH}^{*}, \mathrm{OH}^{*}$ and $\mathrm{O}^{*}$ species were calculated to obtain the values of their corresponding ZPE and TS values. The Gibbs free energy of $\mathrm{O}_{2}$ was derived from $\mathrm{H}_{2} \mathrm{O} \rightarrow \mathrm{H}_{2}+(1 / 2) \mathrm{O}_{2}$ using the experimental reaction energy of $2.46 \mathrm{eV}$ to avoid the error by the DFT calculation in describing the high-spin ground state of the $\mathrm{O}_{2}$ molecule.

\section{Reference}

1. J. K. Nørskov, J. Rossmeisl, A. Logadottir, L. Lindqvist, J. R. Kitchin, T. Bligaard, H. Jonsson, J. Phys. Chem. B, 2004, 108,17886-17892. 


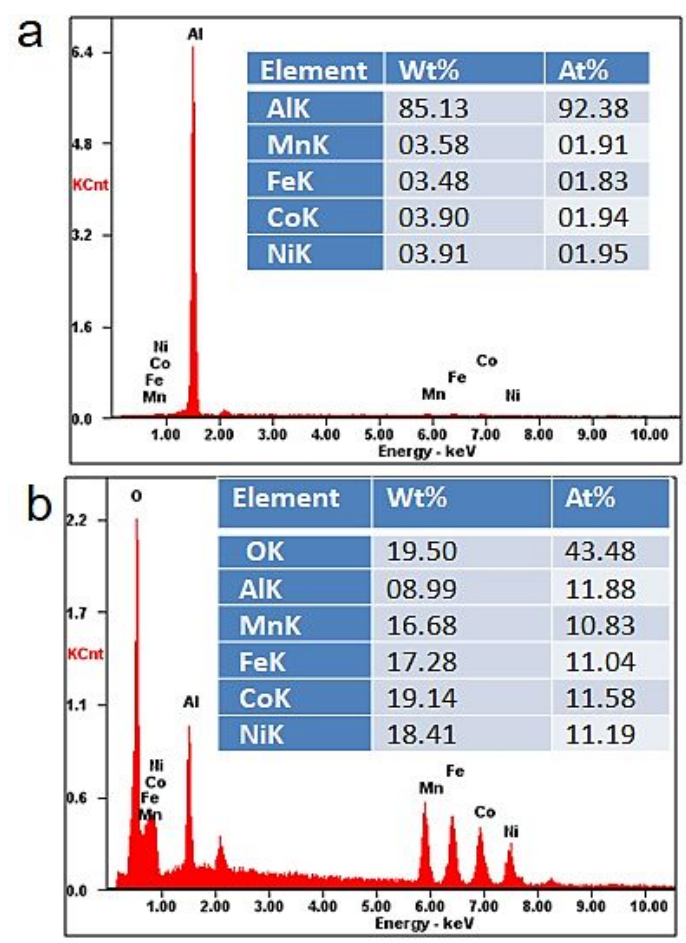

Figure S1. EDS spectra of the AlFeCoNiMn sample before (a) and after (b) dealloying in $0.5 \mathrm{M} \mathrm{NaOH}$ solution. 


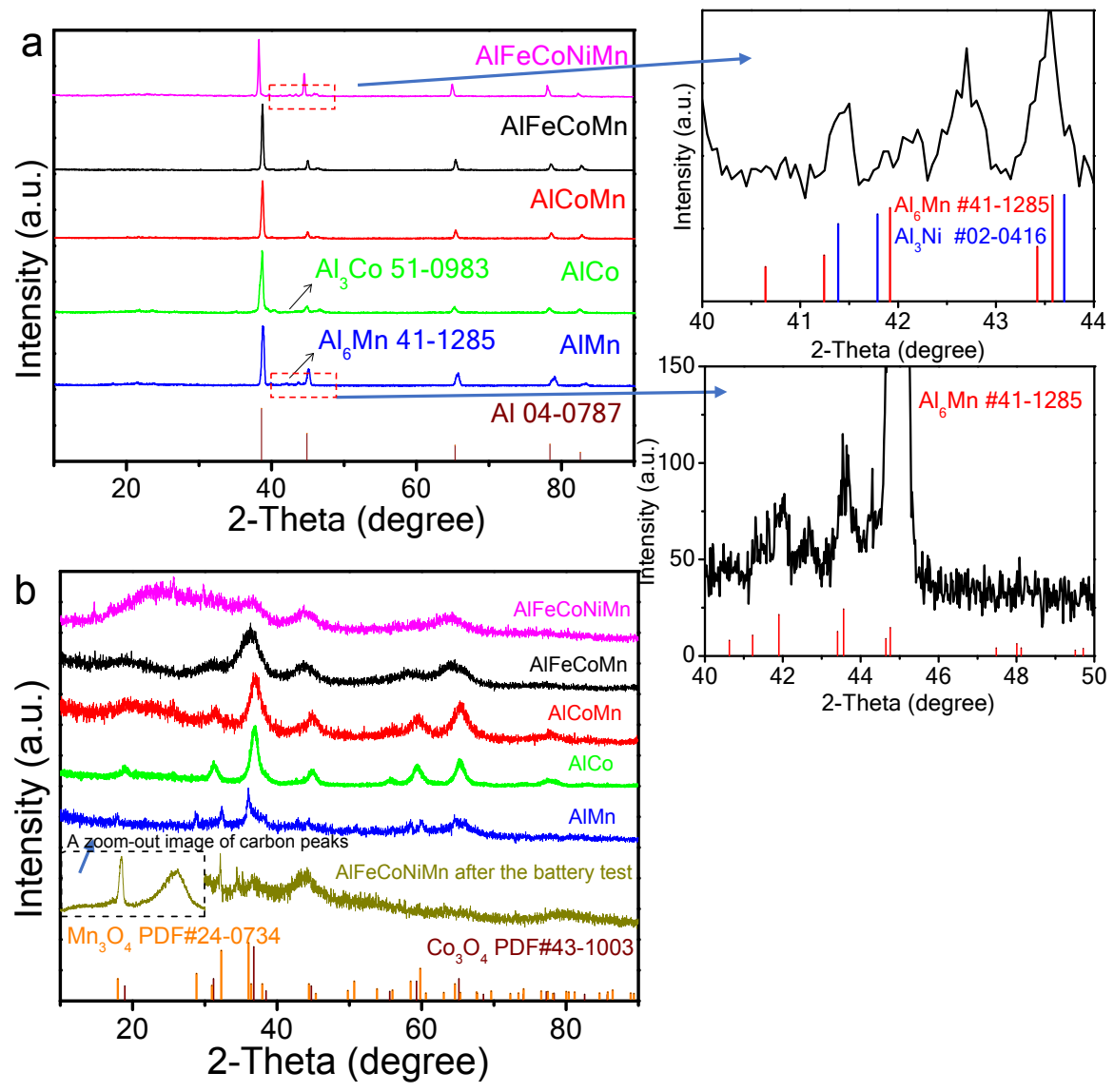

Figure S2. XRD patterns of the precursor alloys before (a) and after (b) dealloying in $0.5 \mathrm{M} \mathrm{NaOH}$ solution. The enlarged partial XRD patterns showing the coexistence of pure $\mathrm{Al}$ phase, $\mathrm{Al}_{3} \mathrm{Ni}$-structrued phase and $\mathrm{Al}_{6} \mathrm{Mn}$-structured phase of $\mathrm{AlFeCoNiMn}$ sample and the presence of $\mathrm{Al}_{6} \mathrm{Mn}$-structured phase in the AlMn sample. The XRD patterns of the dealloyed AlFeCoNiMn after battery test was also included in (b). It shows that phase structure of spinel oxides is not changed. Due to the addition of carbon in the electrocatalyst, high intensity carbon peaks can also be observed. 


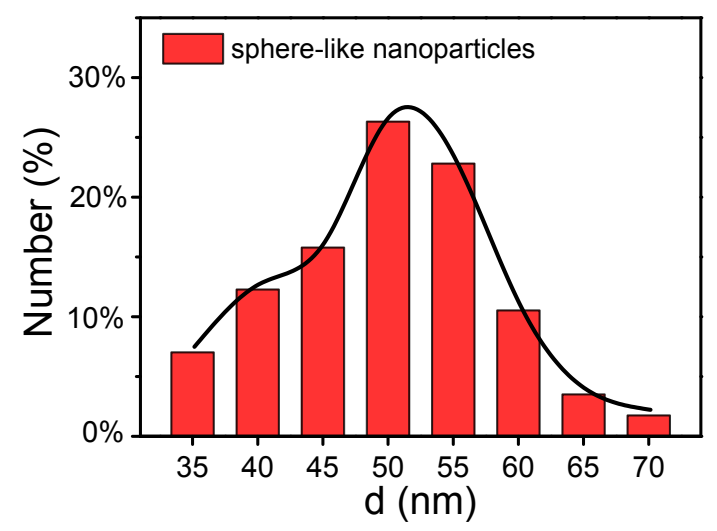

b

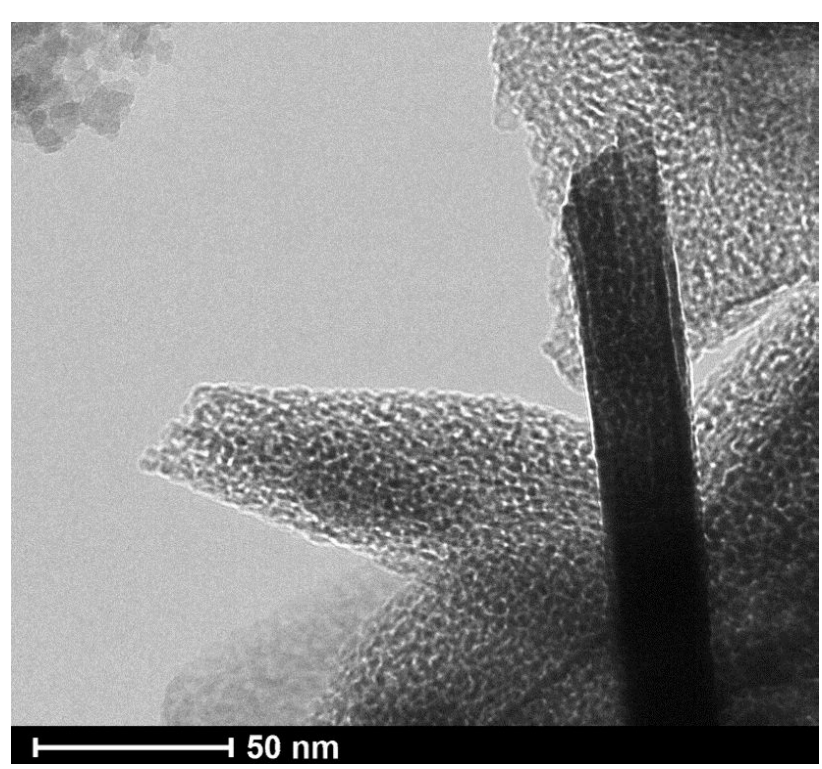


C
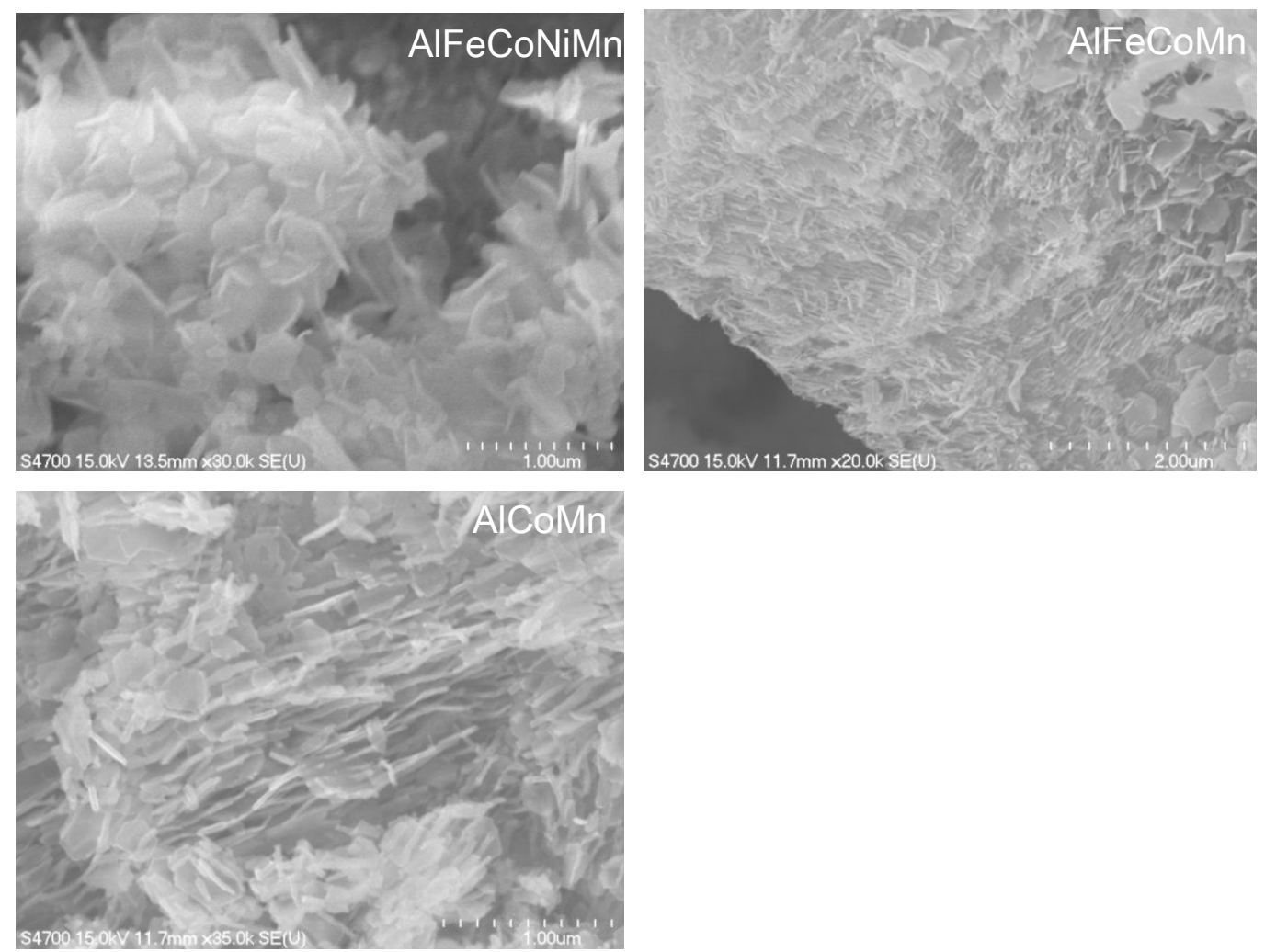

Figure S3. Size distribution of the spheres or elongated sphere-like $(\mathrm{FeCoNi})_{3} \mathrm{O}_{4}$ nanoparticles (a, 100 NPs are measured), TEM image of the $\mathrm{Mn}_{3} \mathrm{O}_{4}$ nanosheets (b) and SEM images of the three dealloyed samples (c).
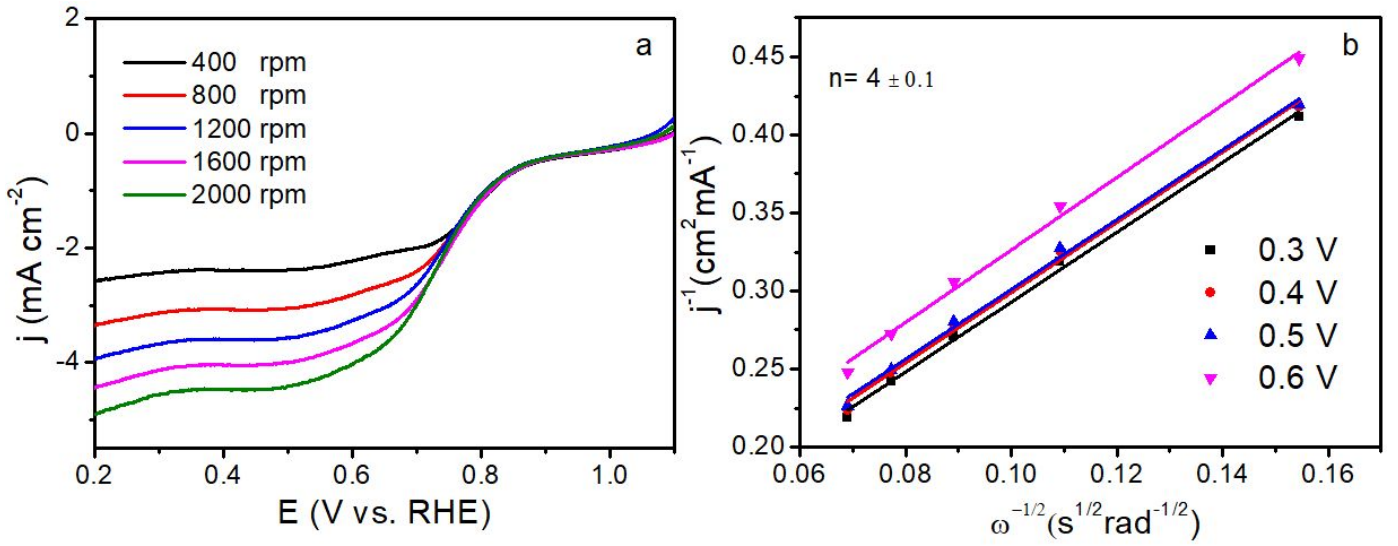

Figure S4. ORR polarization curves of the $\mathrm{np}-\mathrm{AlFeCoNiMn}$ at various rotation rates (a) and the Koutecky-Levich plots at different potentials (b). 

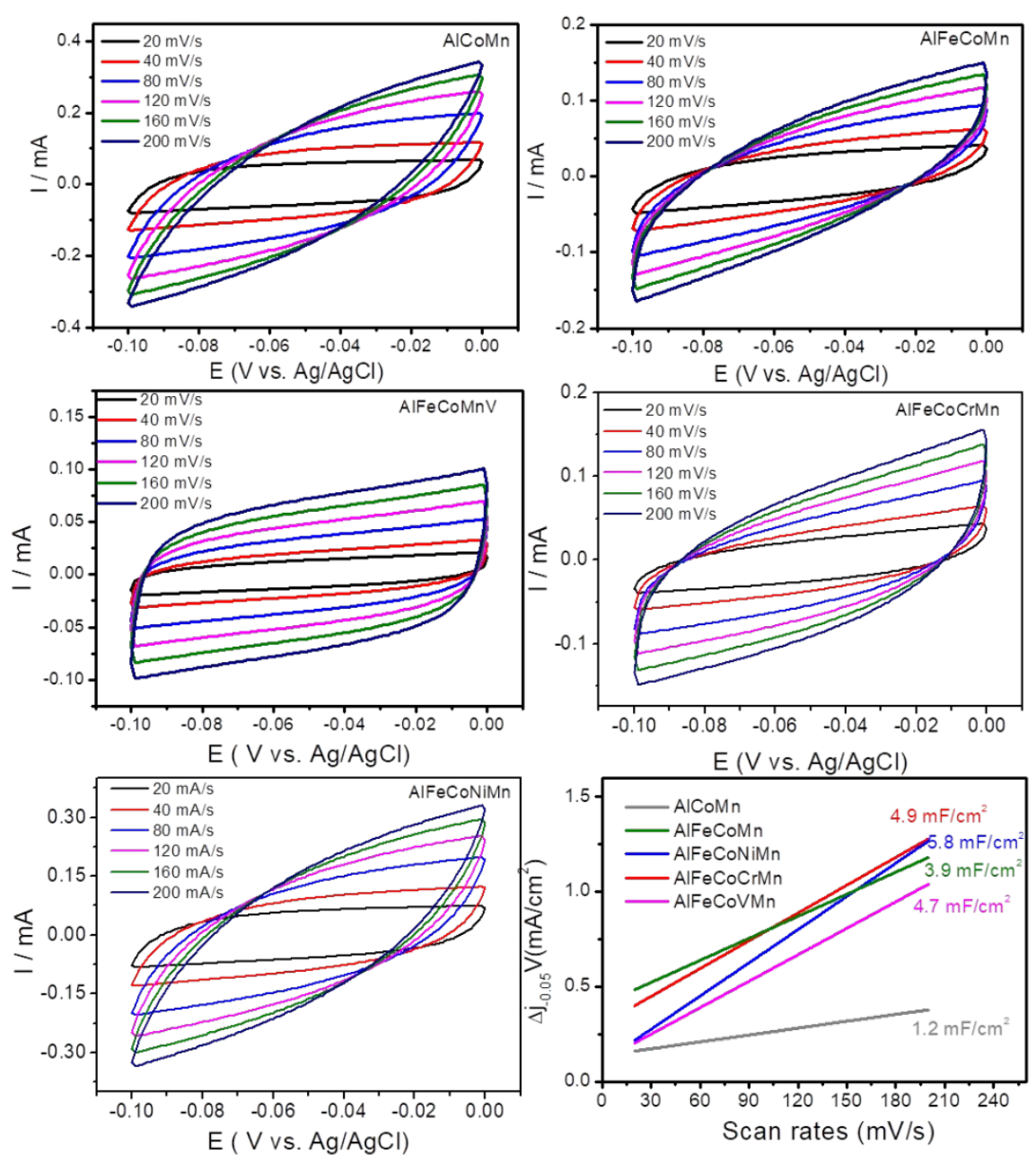

Figure S5. CV curves of these dealloyed samples showing the doule layer capacitance without electrochemcial redox reactions and the difference in current density $(\Delta \mathrm{J}=\mathrm{Ja}-\mathrm{Jc})$ at $-0.05 \mathrm{~V}(\mathrm{vs} . \mathrm{Ag} / \mathrm{AgCl})$ plotted against scan rates and fitted to a linear regression for the estimation of capacitance (last figure). 


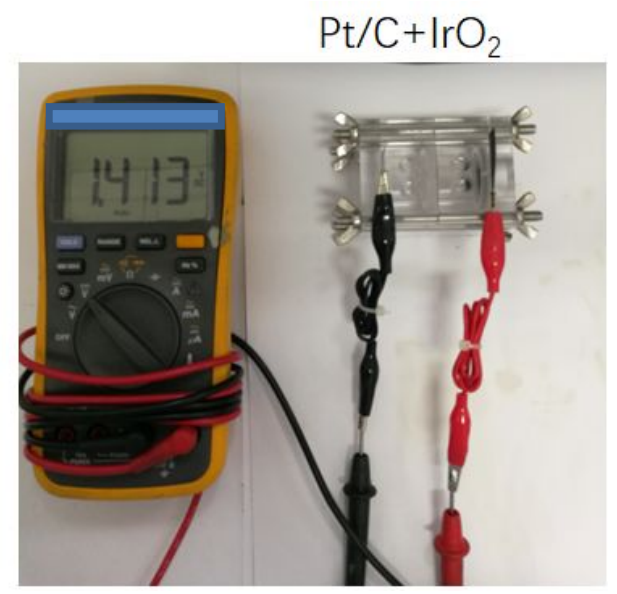

Figure S6. Open-circuit potentials of the liquid $\mathrm{Zn}$-air batteries based on $\mathrm{Pt} / \mathrm{C}+\mathrm{IrO}_{2}$ catalysts.

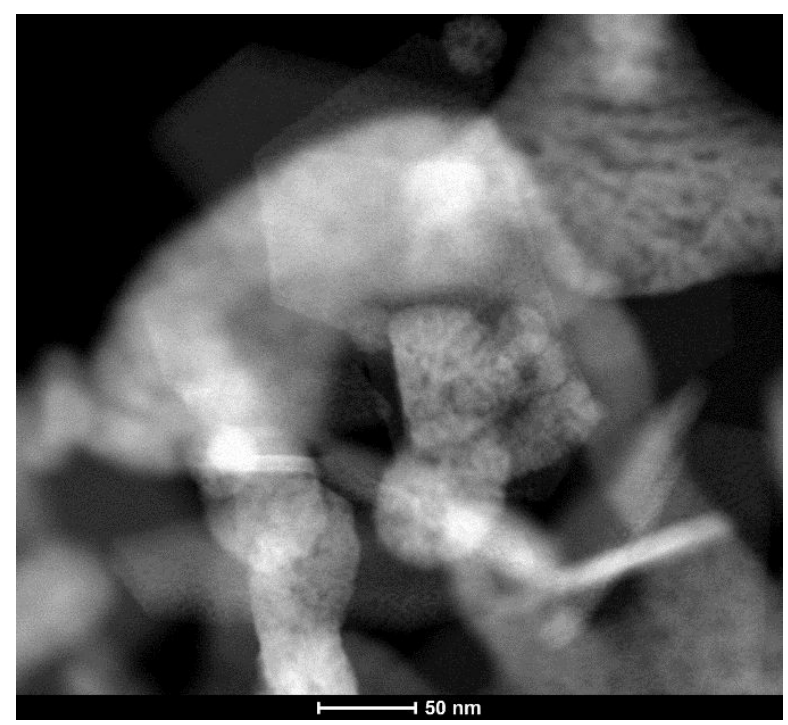

Figure S7. STEM image of the dealloyed AlFeCoNiMn after the battery test. 
a

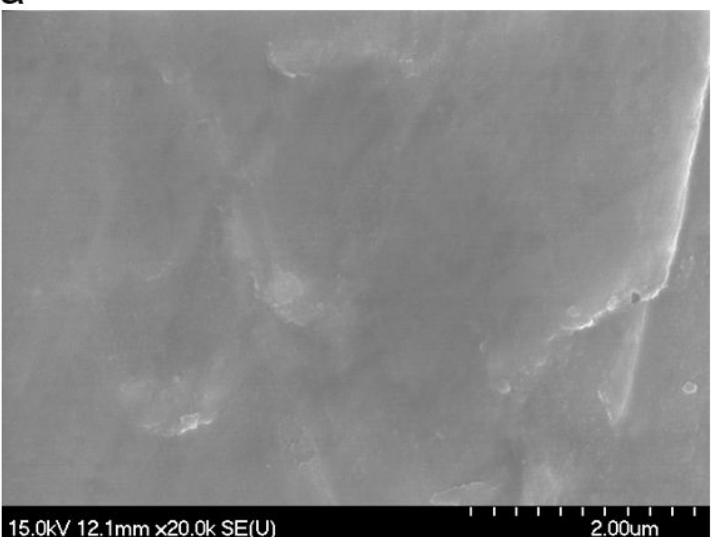

$15.0 \mathrm{kV} 12.1 \mathrm{~mm} \times 20.0 \mathrm{k}$ SE(U) b

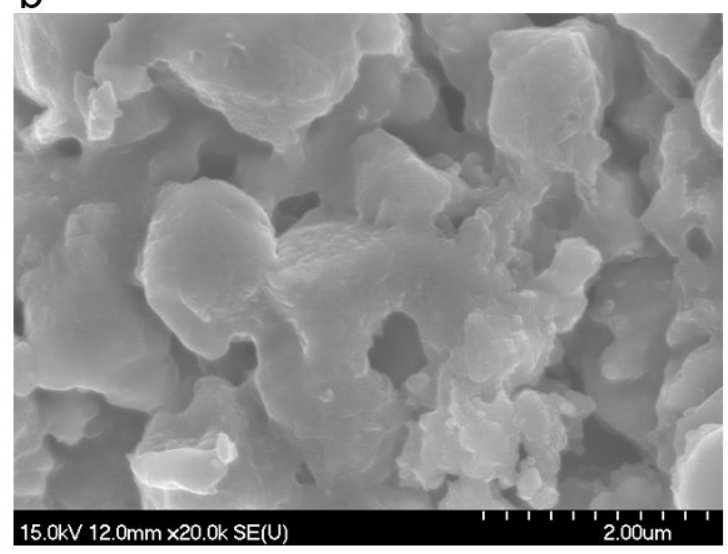

Figure S8. SEM images of the Zn electrode before (a) and after (b) the battery test. 
Table S1. Comparison of the OER performance with literature data.

\begin{tabular}{|c|c|c|c|c|c|}
\hline Catalysts & Substrate & Electrolyte & $\begin{array}{c}\eta(\mathrm{mV}) \\
\text { at } \mathrm{j}=10 \mathrm{~mA} \\
\mathrm{~cm}^{-2}\end{array}$ & $\begin{array}{l}\text { Tafel slope } \\
\left(\mathrm{mV} \mathrm{dec}{ }^{-1}\right)\end{array}$ & Reference \\
\hline $\mathrm{NiFe} \mathrm{LDH}$ & GCE & $1.0 \mathrm{M} \mathrm{KOH}$ & $300 *$ & 40 & $\begin{array}{c}\text { Nat Commun 2014, } \\
5.4477\end{array}$ \\
\hline $\mathrm{Ni}_{\mathrm{x}} \mathrm{Co}_{3-\mathrm{x}} \mathrm{O}_{4} \mathrm{NW}$ & GCE & $1.0 \mathrm{M} \mathrm{KOH}$ & $370 *$ & $59-64$ & $\begin{array}{c}\text { Adv Mater 2010 } \\
22,1926\end{array}$ \\
\hline CoP-CNT & GCE & $0.1 \mathrm{M} \mathrm{NaOH}$ & $330 *$ & 50 & $\begin{array}{l}\text { Acs Appl Mater } \\
\text { Inter 2015, } 7 \text {, } \\
28412\end{array}$ \\
\hline $\mathrm{NiFe} \mathrm{NO}_{3}$ & Nickel foam & $0.1 \mathrm{M} \mathrm{KOH}$ & 270 & 56 & $\begin{array}{l}\text { Angew. Chem. Int. } \\
\text { Ed., 2017,56,11258 }\end{array}$ \\
\hline $\mathrm{NiO} / \mathrm{Ni}$ & Nickel foam & $1.0 \mathrm{M} \mathrm{KOH}$ & 345 & 53 & $\begin{array}{l}\text { J. Mater. Chem. A, } \\
\text { 2016, 4, } 9797\end{array}$ \\
\hline CoFe LDHs-Ar & Nickel foam & $1.0 \mathrm{M} \mathrm{KOH}$ & 266 & 38 & $\begin{array}{l}\text { Angew. Chem. Int. } \\
\text { Ed., 2017,56,5867 }\end{array}$ \\
\hline $\mathrm{Ni}_{2} \mathrm{P}$ NPs & Nickel foam & $1.0 \mathrm{M} \mathrm{KOH}$ & $290 *$ & 47 & $\begin{array}{c}\text { Energy Environ Sci } \\
\mathbf{2 0 1 5}, 8,2347\end{array}$ \\
\hline CoMn LDH & GCE & $1.0 \mathrm{M} \mathrm{KOH}$ & 324 & 43 & $\begin{array}{c}\text { J. Am. Chem. Soc. } \\
\text { 2014, } 136 \\
16481-16484 .\end{array}$ \\
\hline G-FeCoW & GCE & $1.0 \mathrm{M} \mathrm{KOH}$ & 223 & 37 & $\begin{array}{c}\text { Science 2016, 352 } \\
\text { 333-337 }\end{array}$ \\
\hline $\mathrm{Co}-\mathrm{Mo}_{2} \mathrm{~N}$ & GCE & $1.0 \mathrm{M} \mathrm{KOH}$ & 302 & 90 & $\begin{array}{c}\text { J. Mater. Chem. A, } \\
\text { 2018, 6, } \\
\text { 20100-20109 }\end{array}$ \\
\hline $\mathrm{Ni}_{3} \mathrm{FeN}$ & Nickel foam & $1.0 \mathrm{M} \mathrm{KOH}$ & 280 & 46 & $\begin{array}{c}\text { Adv. Energy. Mater } \\
\mathbf{2 0 1 6}, 6,1502333\end{array}$ \\
\hline $\mathrm{Au} / \mathrm{NiFe} \mathrm{LDH}$ & GCE & $1.0 \mathrm{M} \mathrm{KOH}$ & 237 & 35 & $\begin{array}{c}\text { J. Am. Chem. Soc. } \\
\text { 2018, } 140 \\
3876-3879\end{array}$ \\
\hline $\mathrm{NiC} / \mathrm{C}$ & GCE & $1.0 \mathrm{M} \mathrm{KOH}$ & 316 & 46 & $\begin{array}{c}\text { Adv. Mater 2016 } \\
28,3326\end{array}$ \\
\hline $\mathrm{Ni}(\mathrm{OH})_{2}$ & GCE & $1.0 \mathrm{M} \mathrm{KOH}$ & $331 *$ & 42 & $\begin{array}{c}\text { J. Am. Chem. Soc. } \\
\text { 2014, 136, } \\
\text { 7077-7084 }\end{array}$ \\
\hline $\mathrm{NiCoP} / \mathrm{C}$ & GCE & $0.1 \mathrm{M} \mathrm{KOH}$ & 330 & 96 & $\begin{array}{l}\text { Angew. Chem. Int. } \\
\text { Ed., 2017, 129, } \\
\text { 3955-3958 }\end{array}$ \\
\hline AlFeCoNiMn & GCE & 1.0М КОН & 290 & 51 & This work \\
\hline AlFeCoCrMn & GCE & $1.0 \mathrm{M}$ КОН & 250 & 44 & This work \\
\hline
\end{tabular}


Table S2. Comparison of the ORR/OER performances with reported values.

\begin{tabular}{|c|c|c|c|c|}
\hline Catalysts & ORR: $E_{1 / 2}(V)$ & OER: $E_{j=10}(V)$ & $\begin{array}{c}\text { Voltage gap: } \Delta \mathrm{E}(\mathrm{V}) \\
\text { (OER-ORR) }\end{array}$ & Reference \\
\hline $\begin{array}{c}\mathrm{Mn}_{3} \mathrm{O}_{4} @ \mathrm{CoMn}_{2} \mathrm{O}_{4}-\mathrm{CoO} \\
\mathrm{NPs}\end{array}$ & 0.83 & 1.68 & 0.85 & $\begin{array}{l}\text { ACS Appl. Mater. Interfaces 2016, 8, } \\
\qquad 17435-17444 .\end{array}$ \\
\hline $\mathrm{c}-\mathrm{CoMn}_{2} / \mathrm{C}$ & 0.82 & 1.78 & 0.96 & Nat. Commun. 2015, 6, 7345. \\
\hline Fe-doped $\mathrm{MnO}_{2}$ & 0.72 & 1.89 & 1.17 & Catal. Sci. Technol. 2019, 9, 1245. \\
\hline Mn-doped $\mathrm{Co}_{3} \mathrm{O}_{4}$ & 0.81 & 1.65 & 0.84 & $\begin{array}{l}\text { ACS Appl. Mater. Interfaces 2016, 8, } \\
20802 .\end{array}$ \\
\hline $\mathrm{Mn}_{0.5}\left(\mathrm{Fe}_{0.3} \mathrm{Ni}_{0.7}\right)_{0.5} \mathrm{O}_{\mathrm{X}}$ & 0.81 & 1.57 & 0.76 & Adv. Funct. Mater. 2020, 30, 1905992. \\
\hline $\mathrm{CoMn}_{2} \mathrm{O}_{4} / \mathrm{N}-\mathrm{rGO}$ & 0.80 & 1.66 & 0.86 & Electrochem. Commun. 2014, 41, 59. \\
\hline CoMnNi-231 & 0.75 & 1.63 & 0.88 & J. Power Sources 2019, 435, 226761. \\
\hline $\begin{array}{l}\mathrm{La}_{1.5} \mathrm{Sr}_{0.5} \mathrm{Ni}_{0.5} \mathrm{Mn}_{0.5} \\
\quad \mathrm{~N}_{\mathrm{i} 0.5} \mathrm{Ru}_{0.5} \mathrm{O}_{6} / \mathrm{C}\end{array}$ & 0.73 & 1.66 & 0.93 & $\begin{array}{l}\text { ACS Appl. Mater. Interfaces 2019, 11, } \\
\text { 21454-21464. }\end{array}$ \\
\hline $\mathrm{NCNT} / \mathrm{Ni}-\mathrm{NiMn}_{2} \mathrm{O}_{4} *$ & 0.71 & 1.53 & 0.82 & $\begin{array}{l}\text { Catal. Sci. Technol. 2019, 9, } \\
\text { 1595-1601 }\end{array}$ \\
\hline $\mathrm{Co}_{0.5} \mathrm{Ni}_{0.5} \mathrm{Mn}_{2} \mathrm{O}_{4}$ & 0.65 & 1.72 & 1.07 & Batteries \& Supercaps. 2020, 3, 1-8 \\
\hline AlFeCoNiMn & 0.77 & 1.52 & 0.75 & This work \\
\hline AlFeCoCrMn & 0.70 & 1.48 & 0.78 & This work \\
\hline
\end{tabular}

*The value of potential was directly estimated from LSVs of the OER current density at $10 \mathrm{~mA} \mathrm{~cm}^{-2}$. 
Table S3. Zero-point energy (ZPE) and entropy correction (TS) at $\mathrm{T}=298 \mathrm{~K}$ for relevant species.

\begin{tabular}{ccc}
\hline Species & ZPE(eV) & TS(eV) \\
\hline $\mathrm{H}_{2} \mathrm{O}$ & 0.56 & 0.67 \\
$\mathrm{H}_{2}$ & 0.27 & 0.41 \\
$\mathrm{OOH}^{*}$ & 0.35 & 0 \\
$\mathrm{OH}^{*}$ & 0.31 & 0.01 \\
$\mathrm{O}^{*}$ & 0.05 & 0 \\
\hline
\end{tabular}


Table S4. Comparison of the liquid Zn-air battery performance with literature data.

\begin{tabular}{|c|c|c|c|c|}
\hline Catalysts & $\begin{array}{l}\text { Open circuit } \\
\text { potential (V) }\end{array}$ & $\begin{array}{c}\text { Peak power } \\
\text { density (mW } \\
\left.\mathbf{c m}^{-2}\right)\end{array}$ & Stability & Ref. \\
\hline Co-N,B-CSs & 1.43 & 100.4 & $14 \mathrm{~h}(128$ cycles $)$ & ACS Nano. 2018, 12, 1894-1901 \\
\hline N,S-CC & 1.36 & 42 & $\begin{array}{l}600 \text { s/cycle } \\
1020 \text { cycles }\end{array}$ & Adv. Sci. 2018, 5, 1800760 \\
\hline N-GCNT/FeCo-3 & 1.48 & 89.3 & $40 \mathrm{~h}$ (240 cycles) & Adv. Energy Mater. 2017, 7, 1602420 \\
\hline $\mathrm{Fe} \mathrm{SAs} / \mathrm{N}-\mathrm{C}$ & -- & 225 & $260 \mathrm{~h}$ & ACS Catal. 2019, 9, 2158-2163 \\
\hline Fe SAs/MC(950) & 1.52 & & $100 \mathrm{~h}$ & ACS Energy Lett. 2018, 3, 2383-2389 \\
\hline NGM-Co & -- & 152 & $60 \mathrm{~h}$ & Adv. Mater. 2017, 29, 1703185 \\
\hline$(\mathrm{Zn}, \mathrm{Co}) / \mathrm{NSC}$ & 1.5 & 150 & -- & Nano Energy. 2019, 58, 277-283 \\
\hline $\mathrm{Cu} @ \mathrm{Fe}-\mathrm{N}-\mathrm{C}$ & 1.48 & 92 & $50000 \mathrm{~s}$ & Adv. Funct. Mater. 2018, 28, 1802596 \\
\hline $\mathrm{S}-\mathrm{C}_{2} \mathrm{NA}$ & 1.49 & 209 & $750 \mathrm{~h}$ & ACS Nano 2018, 12, 596-608 \\
\hline $\mathrm{Co}(\mathrm{OH})_{2} / \mathrm{CoPt} / \mathrm{NCN}$ & -- & 171 & 300 cycles & $\begin{array}{l}\text { ACS Appl. Mater. Interfaces 2019, 11, } \\
\text { 4983-4994 }\end{array}$ \\
\hline $\mathrm{CoSAs@NC}$ & 1.46 & 105.3 & $1000 \mathrm{~min}$ & Angew. Chem. Int. Ed. 2019, 58, 1-7 \\
\hline $\mathrm{mPtPd}-\mathrm{NF}$ & 1.52 & 111 & -- & $\begin{array}{l}\text { ACS Sustainable Chem. Eng. 2018, 6, } \\
\text { 12367-12374 }\end{array}$ \\
\hline SilkNC/KB & 1.426 & 91.2 & $33 \mathrm{~h}$ (100 cycles $)$ & Chem. Mater. 2019, 31, 3, 1023-1029 \\
\hline $\mathrm{CoO}_{\mathrm{x}} @ \mathrm{NGCR}$ & 1.40 & 90.1 & $1000 \mathrm{~min}$ & $\begin{array}{c}\text { ACS Sustainable Chem. Eng. 2018, 6, } \\
\text { 15811-15821 }\end{array}$ \\
\hline $\begin{array}{c}\mathrm{N}-\mathrm{CoSe}_{2} / 3 \mathrm{D} \\
\mathrm{Ti}_{3} \mathrm{C}_{2} \mathrm{~T}_{\mathrm{x}}\end{array}$ & 1.43 & 142 & $166 \mathrm{~h}(500$ cycles $)$ & ACS Materials Lett. 2019, 1, 432- 439 \\
\hline $\begin{array}{c}\text { CoSx@Cu2MoS4- } \\
\text { MoS2/NSG }\end{array}$ & 1.44 & 40 & $5.5 \mathrm{~h}$ & Adv. Energy Mater. 2020, 10, 1903289 \\
\hline CoNi@NCNT/NF & 1.40 & 127 & $90 \mathrm{~h}$ & Adv. Energy Mater. 2018, 8, 1800480 \\
\hline np-AlFeCoNiCr & 1.55 & 128 & $120 \mathrm{~h}$ & $\begin{array}{c}\text { Appl. Catalysis B: Environ. 2020, 268, } \\
118431\end{array}$ \\
\hline
\end{tabular}


